\title{
A New Analytic Method of Cold Standby System Reliability Model with Priority
}

\author{
Di Peng,Ni Zichun and $\mathrm{Hu}$ Bin
}

Naval University of Engineering, Wuhan 430000, China

\begin{abstract}
For different importance of components in equipment system, a cold standby system with two different components is studied when important components enjoy the priority in use and maintenance. Considering the application of exponential distribution, Weibull distribution and other typical distributions in resolving the problems subject to complicated calculation and strict constraints in the past reliability modelling, the highly applicable phase-type (PH) distribution is utilized to describe the life and maintenance time of system components in a unified manner. A system reliability model is built for wider applicability. With the matrix analysis method, expressions are obtained for a number of reliability indicators such as system reliability function, steady-state availability, mean up time and mean down time of system. In the end, examples are presented to verify the correctness and applicability of the model.
\end{abstract}

\section{Introduction}

In the research and development of equipment system, the "one service one standby" cold standby structure is often utilized to improve system reliability, enhance equipment availability, and lower system cost. Considering different system structures, importance and service and repair costs of different components, it is of practical significance to determine the priority of key and important components in use and maintenance to some extent. Hence, the cold standby repairable system with priority in use and maintenance [1], as a classical model, is widely applied in the practical engineering.

Zhang [2] studied the cold standby system subject to incomplete maintenance and with priority in use. Using the complete maintenance model and geometric process maintenance model, Leung [3] explored the conditions with priority in maintenance. Cao and Cheng [4] studied two-component repairable system with priority in use and maintenance and its life and maintenance time subject to exponential distribution, and obtained a number of reliability indicators. Osaki et al. [5] employed the Markov renewal theory to study a reliability model for the cold standby system with priority in use and maintenance, which had the service time and maintenance time of components with priority subject to general distribution, but the service time and maintenance time of the components without priority subject to exponential distribution.

In the past studies on reliability, it is often assumed that the service time and maintenance time of each component in a system are subject to exponential distribution, normal distribution and other typical distributions. However, many cold standby systems contain different types of component in the practical engineering, and the lives of these components follow different laws, so the corresponding maintenance activities are different. If exponential distribution, normal distribution and other typical distributions are utilized to describe the parameters of system in the reliability modelling, it will be more difficult to analyze the model.

To enhance the applicability of model, and retain the feature that exponential distribution is easy to calculate, Netus [6] put forth the PH distribution and applied it in the reliability modeling in 1975 . As $\mathrm{PH}$ distribution can satisfactorily fit the experimental data and complicated distribution of reliability engineering, has very good analytic feature, and widen the application range of model, it has been widely applied in the reliability field. Faddy [7] utilized PH distribution to represent the service time of components, and obtained the reliability law of singlecomponent system through renewal process.

Based on the above studies, this paper introduces the $\mathrm{PH}$ distribution to describe the life distribution and maintenance time of components, so as to address such problems as too strict assumptions for the reliability model of the cold standby system with two different components and priority in use and maintenance, as well as poor applicability of the model. A more applicable reliability model is built to obtain the analytic expressions for a number of reliability indicators including system transient-state availability, reliability, and failure frequency, steady-state availability and failure frequency, as well as mean up time, mean down time and mean cycle time of system.

* Corresponding author: $2477433853 @$ qq.com 


\section{System Description and Assumption}

It is assumed that it is a cold standby system with two components of different types and one unit of maintenance equipment. The assumption is detailed as follows:

1) At the beginning of the system, two components start operation in a good state. Component 1 starts operation first, and component 2 is in standby state;

2) The life of Component 1 is subject to $m$-order $\mathrm{PH}$ distribution, which is represented by $(\boldsymbol{\alpha}, \boldsymbol{T})$;

3) The life of Component 2 is subject to $k$-order $\mathrm{PH}$ distribution, which is represented by $(\gamma, L)$;

4) Component 1 has priority over Component 2. In other words, Component 1 has priority in use and maintenance. When Component 1 is satisfactorily repaired, if Component 2 is in service state, Component 2 is replaced by it, so Component 1 enters the service state, and Component 2 enters the standby state. When Component 2 is in maintenance state, if Component 1 fails, Component 2 transits from the maintenance state into the state of waiting for maintenance, so maintenance equipment starts repairing Component 1 ;

5) After being repaired by maintenance equipment, components are completely repaired. Among them, the maintenance time of Component 1 is subject to the $n$ order $\mathrm{PH}$ distribution, and denoted by $(\boldsymbol{\beta}, \boldsymbol{S})$. The maintenance time of Component 2 is also subject to $g$ order PH distribution, and represented by $(\boldsymbol{\theta}, \boldsymbol{U})$;

6) All the above random variables are independent from each other;

7) The time taken to replace failed component with standby component is ignored.

\section{Model Construction}

\subsection{System State Space}

$K(t)$ stands for the number of failed components in the system, $(K(t)=0,1,2)$. Let $J(t)=\left\{j_{1}(t), j_{2}(t)\right\}$ be the phase at which any intact component in the system is; and let $I(t)=\left\{i_{1}(t), i_{2}(t)\right\}$ be the phase at which maintenance is performed, so $\{K(t), J(t), I(t)\}$ is a multidimensional Markov chain.

The state space of system is represented by $\Omega=\mathrm{H}_{0} \cup \mathrm{H}_{1} \cup \mathrm{H}_{2} \cup \mathrm{H}_{3}$, in which:

$\mathrm{H}_{0}=\left\{0, j_{1}(t)\right\}$ means that there is not any failed component in the system. Component 1 is in service state; Component 2 is in standby state; and Component 1 in service is at the phase $j_{1}(t)$, in which $1 \leq j_{1}(t) \leq m$.

$\mathrm{H}_{1}=\left\{1, j_{1}(t), i_{2}(t)\right\}$ means that the system has 1 failed component; Component 1 in service is at the phase $j_{1}(t)$, and maintenance equipment is repairing at the phase $i_{2}(t)$, in which $1 \leq j_{1}(t) \leq m, 1 \leq i_{2}(t) \leq g$.

$\mathrm{H}_{2}=\left\{1, j_{2}(t), i_{1}(t)\right\}$ means that the system has 1 failed component; Component 2 in service is at the phase $j_{2}(t)$, and maintenance equipment is repairing at the phase $i_{1}(t)$, in which $1 \leq j_{2}(t) \leq k, 1 \leq i_{1}(t) \leq n$.

$\mathrm{H}_{3}=\left\{2, i_{1}(t)\right\}$ means that the system has 2 failed components, so it is in shutdown state; both Components 1 and 2 fail, and maintenance equipment is repairing at the phase $i_{1}(t)$, in which $1 \leq i_{1}(t) \leq n$.

\subsection{State Transition Matrix}

1) $\mathrm{H}_{0}$ internal transition; $K(t)=0$ means that there is not any failed component in the system, and Component 2 is in cold standby state. At this time, there is only the phase transition of Component 1 in service, so it can be denoted by $\boldsymbol{T}$;

2) $\mathrm{H}_{1}$ internal transition; $K(t)=1$ means that there is a failed component in the system, and Component 1 is in service state, while Component 2 is in maintenance state. As the phases of Component 1 in service and maintenance equipment cannot be changed at the same time, their transition matrix can be denoted by $\boldsymbol{T} \oplus \boldsymbol{U}$;

3) $\mathrm{H}_{2}$ internal transition; $K(t)=1$ means that there is 1 failed component in the system, and Component 2 is in service state, while Component 1 is in maintenance state. As the phases of Component 2 in service and maintenance equipment cannot be changed at the same time, their transition matrix can be denoted by $\boldsymbol{L} \oplus \boldsymbol{S}$;

4) $\mathrm{H}_{3}$ internal transition; $K(t)=2$ means that the system has 2 failed components, i.e. Components 1 and 2 are both in maintenance state, so the system fails. At this time, there is only the phase transition of failed Component 1, which can be denoted by $\boldsymbol{S}$;

Similarly, the matrix for transition from macro state $k$ to the state $k+1, k-1$. The infinitesimal generator $Q$ of the Markov chain is obtained as follows:

$$
\boldsymbol{Q}=\left[\begin{array}{cccc}
\boldsymbol{A}_{0,0} & \boldsymbol{O} & \boldsymbol{A}_{0,2} & \boldsymbol{O} \\
\boldsymbol{B}_{1,0} & \boldsymbol{B}_{1,1} & \boldsymbol{O} & \boldsymbol{B}_{1,3} \\
\boldsymbol{C}_{2,0} & \boldsymbol{O} & \boldsymbol{C}_{2,2} & \boldsymbol{C}_{2,3} \\
\boldsymbol{O} & \boldsymbol{D}_{3,1} & \boldsymbol{O} & \boldsymbol{D}_{3,3}
\end{array}\right]
$$

The blocks of matrix $\mathbf{Q}$ are presented as follows:

$$
\begin{aligned}
& \boldsymbol{A}_{0,0}=\boldsymbol{T} \quad \boldsymbol{A}_{0,2}=\boldsymbol{T}^{0} \boldsymbol{\beta} \otimes \boldsymbol{\gamma} \\
& \boldsymbol{B}_{1,0}=\boldsymbol{U}^{0} \otimes \boldsymbol{I} \quad \boldsymbol{B}_{1,1}=\boldsymbol{T} \oplus \boldsymbol{U} \quad \boldsymbol{B}_{1,3}=\boldsymbol{T}^{0} \boldsymbol{\beta} \otimes \boldsymbol{e}_{g} \\
& \boldsymbol{C}_{2,0}=\boldsymbol{S}^{0} \boldsymbol{\alpha} \otimes \boldsymbol{e}_{n} \quad \boldsymbol{C}_{2,2}=\boldsymbol{L} \oplus \boldsymbol{S} \quad \boldsymbol{C}_{2,3}=\boldsymbol{L}^{0} \otimes \boldsymbol{I} \\
& \boldsymbol{D}_{3,1}=\boldsymbol{S}^{0} \boldsymbol{\alpha} \otimes \boldsymbol{\theta} \quad \boldsymbol{D}_{3,3}=\boldsymbol{S}
\end{aligned}
$$




\subsection{System Steady-state Probability Vector}

When the system enters the steady state, the probabilities of states in the infinitesimal generator form a steady-state probability vector $\boldsymbol{\pi}=\left(\boldsymbol{\pi}_{0}, \boldsymbol{\pi}_{1}, \boldsymbol{\pi}_{2}, \boldsymbol{\pi}_{3}\right)$, which satisfies the following equation:

$$
\left\{\begin{array}{l}
\pi Q=0 \\
\pi e=1
\end{array}\right.
$$

The above equation is written into:

$$
\begin{aligned}
& \boldsymbol{\pi}_{0} A_{0,0}+\boldsymbol{\pi}_{1} \boldsymbol{B}_{1,0}+\boldsymbol{\pi}_{2} \boldsymbol{C}_{2,0}=\mathbf{0} \\
& \boldsymbol{\pi}_{1} \boldsymbol{B}_{1,1}+\boldsymbol{\pi}_{3} \boldsymbol{D}_{3,1}=\mathbf{0} \\
& \boldsymbol{\pi}_{0} \boldsymbol{A}_{0,2}+\boldsymbol{\pi}_{2} \boldsymbol{C}_{2,2}=\mathbf{0} \\
& \boldsymbol{\pi}_{1} \boldsymbol{B}_{1,3}+\boldsymbol{\pi}_{2} \boldsymbol{C}_{2,3}+\boldsymbol{\pi}_{3} \boldsymbol{D}_{3,3}=\mathbf{0} \\
& \boldsymbol{\pi}_{0} \boldsymbol{e}+\boldsymbol{\pi}_{1} \boldsymbol{e}+\boldsymbol{\pi}_{2} \boldsymbol{e}+\boldsymbol{\pi}_{3} \boldsymbol{e}=1
\end{aligned}
$$

After solving the above equations, the system steadystate probability vector $\boldsymbol{\pi}_{i}(i=0,1,2,3)$ is calculated.

\section{System Reliability Indicators}

\subsection{System Reliability Function}

System reliability function is the probability of normal operation of the system before the time $t$. For the model in this paper, system reliability refers to the probability that the system is in the state space $\mathrm{H}_{0} \cup \mathrm{H}_{1} \cup \mathrm{H}_{2}$. The failure state $H_{3}$ of the system is combined into the absorptive state. Let $K(t)$ be the number of failed components in the system at the time $t$, i.e. $(K(t)=0,1) ;\{K(t), J(t), I(t)\}$, which constitutes a new multidimensional Markov chain. The corresponding probability transition matrix is as follows:

$$
\boldsymbol{O}=\left[\begin{array}{ccc}
\boldsymbol{A}_{0,0} & \boldsymbol{O} & \boldsymbol{A}_{0,2} \\
\boldsymbol{B}_{1,0} & \boldsymbol{B}_{1,1} & \boldsymbol{O} \\
\boldsymbol{C}_{2,0} & \boldsymbol{O} & \boldsymbol{C}_{2,2}
\end{array}\right]
$$

Let $\mathbf{W}_{i}(t)$ be the probability that the system is in the state $H_{i}$; and $\mathbf{W}_{i}(t)$ and $\boldsymbol{O}$ satisfy the following differential equation:

$$
\left\{\begin{array}{l}
\boldsymbol{W}^{\prime}(t)=\boldsymbol{W}(t) \boldsymbol{O} \\
\boldsymbol{O}(0)=\boldsymbol{\eta}
\end{array}\right.
$$

In which, $\boldsymbol{W}(t)=\left(\boldsymbol{W}_{0}(t), \boldsymbol{W}_{1}(t), \boldsymbol{W}_{2}(t)\right)$;

The differential equation is solved to obtain $R(t)=\boldsymbol{\eta} \exp (\boldsymbol{O} t) \boldsymbol{e}, \quad$ in which $\boldsymbol{\eta}=(\boldsymbol{\alpha}, \boldsymbol{0}, \ldots, \boldsymbol{0}) \quad$ is $1 \times(m+m g+k n)$-order row vector; and $\boldsymbol{e}$ is $(m+m g+k n) \times 1$-order column vector.

\subsection{Mean Time between Failures of System}

Mean time between failures (MTBF) of system refers to the time taken for the system to exit and reenter the failure state. To the model, when the system exits the failure state $\mathrm{H}_{3}$, it operates normally; when the system reenters the state $\mathrm{H}_{3}$, it fails. According to the definition of Phasetype (PH), the MTBF of system is subject to Phase-type $(\mathrm{PH})$, and has $m+m g+k n$-order irreducible expression $(\boldsymbol{\eta}, \boldsymbol{O})$, so the MTBF of system is as follows:

$$
M T B F=-\eta \boldsymbol{O}^{-1} \boldsymbol{e}
$$

\subsection{System Steady-state Availability}

System steady-state availability means the probability that the system is in the state space $\mathrm{H}_{0} \cup \mathrm{H}_{1} \cup \mathrm{H}_{2}$ after longterm operation. The system steady-state availability can be defined as follows:

$$
A=\sum_{i=0}^{2} \pi_{i} e=1-\pi_{3} e
$$

\subsection{System Steady-state Failure Frequency}

System steady-state failure frequency refers to the times that the system enters the failure state within a unit time. To this model, the system can only enter the failure state $\mathrm{H}_{3}$ from the states $\mathrm{H}_{1}$ and $\mathrm{H}_{2}$, so there is:

$$
M=\pi_{1} B_{1,3} e+\pi_{2} C_{2,3} e
$$

The mean up time, mean down time and mean cycle time of the system are as follows:

$$
\begin{aligned}
M U T & =\frac{A}{M} \\
M D T & =\frac{1-A}{M} \\
M C T & =\frac{1}{M}
\end{aligned}
$$

\section{Example Analysis}

In the engineering application, a cold standby system with priority may contain different types of component, and the life distribution of these components may belong to different types of distribution. When a repairman maintains the system, the maintenance time of different components may be subject to different types of distribution as well. In this case, Markov process, Markov renewal process and other methods are not applicable. To prove the correctness of this model and demonstrate the applicability and good analytic performance of the model, two examples are presented as follows: it is first assumed that the lives and maintenance time of components are subject to exponential distribution, and the calculation results are compared with those in Reference [4]. After that, calculation is carried out for the conditions that life and maintenance time are subject to different types of distribution, so as to verify the applicability of the model. 


\subsection{Verification of Correctness}

It is assumed that a cold standby system consists of two different components. Among them, Component 1 enjoys the priority in use and maintenance, while Component 2 is in standby state. The life of Component 1 is subject to the exponential distribution $\lambda_{1}=0.2$, while the life of Component 2 is subject to the exponential distribution $\lambda_{2}=1.2$. The maintenance time of Component 1 and Component 2 is subject to the exponential distribution $\mu_{1}=2, \mu_{2}=3$ respectively.

The infinitesimal generator of the system can be obtained as follows:

$$
\boldsymbol{Q}=\left[\begin{array}{cccc}
-\lambda_{1} & 0 & \lambda_{1} & 0 \\
\mu_{2} & -\lambda_{1}-\mu_{2} & 0 & \lambda_{1} \\
\mu_{1} & 0 & -\lambda_{2}-\mu_{1} & \lambda_{2} \\
0 & \mu_{1} & 0 & -\mu_{1}
\end{array}\right]
$$

The state transition equations of the system are as follows:

$$
\left\{\begin{array}{l}
\frac{d P_{0}(t)}{d t}=-\lambda_{1} P_{0}(t)+\lambda P_{2}(t) \\
\frac{d P_{1}(t)}{d t}=\mu_{2} P_{0}(t)-\left(\lambda_{1}+\mu_{2}\right) P_{1}(t)+\lambda_{1} P_{3}(t) \\
\frac{d P_{2}(t)}{d t}=\mu_{1} P_{0}(t)-\left(\lambda_{2}+\mu_{1}\right) P_{2}(t)+\lambda_{2} P_{3}(t) \\
\frac{d P_{2}(t)}{d t}=\mu_{1} P_{1}(t)-\mu_{1} P_{3}(t)
\end{array}\right.
$$

When $t \rightarrow \infty$, i.e. the system is in steady state, the steady-state probability equations can be obtained as follows:

$$
\left\{\begin{array}{l}
-\lambda_{1} P_{0}+\lambda P_{2}=0 \\
\mu_{2} P_{0}-\left(\lambda_{1}+\mu_{2}\right) P_{1}+\lambda_{1} P_{3}=0 \\
\mu_{1} P_{0}-\left(\lambda_{2}+\mu_{1}\right) P_{2}+\lambda_{2} P_{3}=0 \\
\mu_{1} P_{1}-\mu_{1} P_{3}=0 \\
P_{0}+P_{1}+P_{2}+P_{3}=1
\end{array}\right.
$$

Equation (16) is submitted into Equations (2) to obtain the same result as Equation (18), so the model proposed in this paper certainly obtains the same reliability indicators as those in Reference [4]. The common results obtained using two methods are as follows:

Steady-state availability $\mathrm{A}=0.9086$; mean time between failures of system $M T B F=8.5$; system steadystate failure frequency $M=0.0686$; mean up time of system $M U T=13.2449$; mean down time of system $M D T=1.3324 ;$ mean cycle time of system $M C T=14.5772$.

The relationship between system reliability $R(t)$ and time $t$ is as presented in Fig. 1:

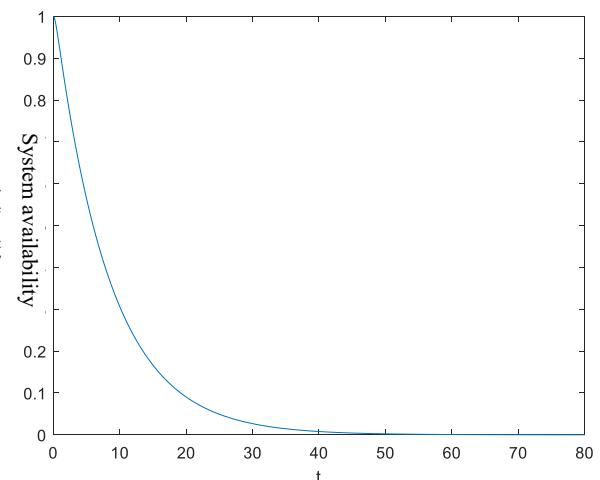

Figure. 1 Example 1 reliability and time relationship

\subsection{Verification of Model Applicability}

It is assumed that a cold standby system consists of two different components and one unit of maintenance equipment. Among them, electronic component 1 has the priority in use and maintenance, and its life distribution is subject to the exponential distribution whose parameter is 0.05 . It can be expressed as PH distribution: $\boldsymbol{\alpha}=(1)$, $\boldsymbol{T}=(-0.05)$. The maintenance time of Component 1 is subject to general distribution, so it can be represented by PH distribution:

$$
\begin{gathered}
\boldsymbol{\beta}=\left(\begin{array}{ll}
1 & 0
\end{array}\right) \\
\boldsymbol{S}=\left(\begin{array}{cc}
-7 & 1 \\
2 & -5
\end{array}\right)
\end{gathered}
$$

Mechanical Component 2 is in cold standby state, and its life distribution is subject to the Weibull distribution whose shape parameter is 3.4 and dimensional parameter is 0.26 . The tool EMpht is employed to express it as $\mathrm{PH}$ distribution:

$$
\begin{aligned}
& \boldsymbol{\gamma}=\left(\begin{array}{lll}
0 & 1 & 0
\end{array}\right) \\
& \boldsymbol{L}=\left(\begin{array}{lcr}
-0.8682 & 0 & 0 \\
0 & -0.8682 & 0.8682 \\
0.8682 & 0 & -0.8682
\end{array}\right)
\end{aligned}
$$

The maintenance time of Component 2 is subject to the Weibull distribution whose shape parameter is 4 and dimensional parameter is 1.5. Its expression as $\mathrm{PH}$ distribution is as follows:

$$
\boldsymbol{U}=\left(\begin{array}{lcc}
-5.7572 & 0 & 0 \\
0 & -5.7572 & 5.7572 \\
5.7572 & 0 & -5.7572
\end{array}\right)
$$

Based on the conclusions in Section 3 and Section 4, the results are obtained when the system is in steady state: system steady-state availability $\mathrm{A}=0.9948$; mean time between failures of system $M T B F=171.3969$; system steady-state failure frequency $M=0.0060$; mean up time of system $M U T=165.8000$; mean down time of system $M D T=0.8667$; mean cycle time of system $M C T=166.6667$. 
The relationship between system reliability $R(t)$ and time $t$ is as presented in Fig. 2:

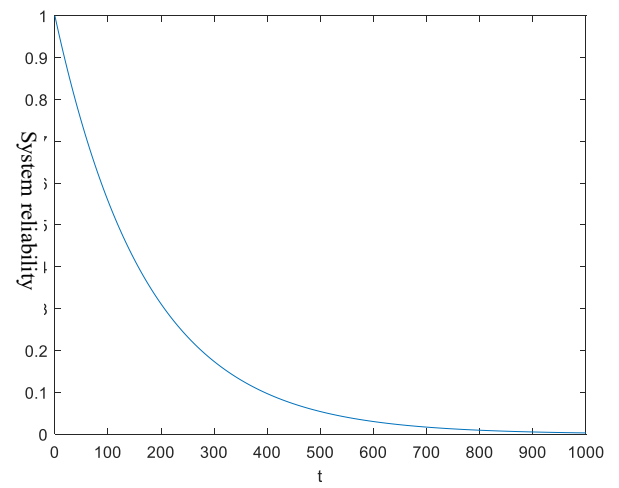

Figure. 2 Example 2 reliability and time relationship

Through the analysis on the results in this example, it is explained that this model can be adjusted to the inputs for different types of distribution, so it has very strong analytic ability. Hence, the application of model is greatly improved.

\section{Conclusion}

This paper mainly studies the cold standby system that consists of two different components and one unit of maintenance equipment, and has important components with priority in use and maintenance. Utilizing the density and good analytic ability of $\mathrm{PH}$ distribution, a more applicable reliability model is built to obtain a number of reliability indicators. Examples are presented to verify the correctness and applicability of the model, which has good value of application in the practical engineering.

\section{References}

1. Y Lam: A maintenance model for two-unit redundant system. Microelectronics and Reliability. J. 37, 497504(1997)

2. Y. L. Zhang and G. J. Wang: A deteriorating cold standby repairable system with priority in use. European Journal of Operational Research. J. 183, 278-295(2007)

3. K. N. F Leung, Y. L. Zhang and K. K. Lai: Analysis for a two dissimilar-component cold standby repairable system with repair priority. Reliability Engineering and System Safety. J. 96, 15421551(2011)

4. J. H. Cao and K. Chen: An introduction to mathematical of reliability. M. 2006

5. T. Nakagawa and S. Osaki: Stochastic behaviour of a two-unit priority standby redundant system with repair. Microelectronics and Reliability. J. 14, 309313(1975)

6. M. F. Neuts and K.S. Meier: On the use of phase type distributions in reliability modelling of systems with two components. OR Spectrum. J. 2, 227-234(1981)
7. M. J. Faddy:Phase-type distributions for failure times. Mathematical and computer modelling. J. 22, 10-12(1995) 